\title{
Cesarean Section and Prophylactic Antibiotics
}

\author{
Shamna.M.S ${ }^{1}$, V.K.Kalaichelvan ${ }^{2}$, Y.M.Fazil Marickar ${ }^{3}$, Deepu.S ${ }^{4}$ \\ ${ }^{I}$ (Department of Pharmacy Practice, Mar Dioscorus college of Pharmacy, Trivandrum, India) \\ ${ }^{2}$ (Department of Pharmacy, Annamalai University, Annamalai Nagar, TamilNadu, India) \\ ${ }_{3}^{3}$ (Department of Surgery, Mount Zion Medical College, Adoor, Pathanamthitta, India) \\ ${ }^{4}$ (Department of Pharmaceutics, Mar Dioscorus college of Pharmacy, Trivandrum, India)
}

\begin{abstract}
Infectious morbidity remains a leading cause of postoperative complications following caesarean delivery. The major infectious complications of caesarean delivery are fever, wound infection, endometritis and urinary tract infection. Postoperative surgical site infection remains a major source of illness and a less frequent cause of death in the surgical patient. The administration of prophylactic antibiotics with in specific interval has been shown to reduce the burden of surgical site infection, but adherence to proper timing guidelines remains problematic. Judicious use of antibiotics in the hospital through effective antibiotic policy and guideline development is then essential.
\end{abstract}

Keywords: Cesarean section, Postcaesarean infectious complication, Prophylactic antibiotics, Surgical antibiotic prophylaxis, Surgical antimicrobial prophylaxis.

\section{Introduction}

Caesarean section is a major operation, with great potential benefit, but also with substantial risks for both mother and baby ${ }^{1}$.Maternal mortality and morbidity are higher than for vaginal delivery, although rates are becoming lower with advances in technology ${ }^{2}$. Infectious morbidity remains a leading cause of postoperative complications following caesarean delivery ${ }^{3}$. A Cesarean section is indicated when delivery is required and cannot be performed vaginally because it will take too long or because it will endanger the mother or the foetus. Cephalopelvic disproportion, previous cesarean, fetal distress, cord prolapsed etc are some common indications of cesarean delivery. The major infectious complications of caesarean delivery are Fever, wound infection, endometritis and UTI(Urinary Tract Infection) ${ }^{4}$

\section{Pathogenesis of post-cesarean infection:}

Postoperative endometritis is a polymicrobial infection caused by bacteria normally present in the host's lower genital tract. The major microorganisms responsible for endometritis are aerobic gram-negative bacilli, principally E.coli; anaerobic gram-negative bacilli, principally Bacteroides species and gardnerella vaginalis; aerobic gram-positive cocci, primarily Group B and Group D streptococci; and anaerobic grampositive cocci, specifically peptococcus species and peptostreptococcus species. ${ }^{5}$ The development of clinical infection is dependent on a complex balance between host defence mechanisms and bacterial virulence factors.

Cesarean delivery alters this balance so as to predispose the patient to infection. During labor and abdominal delivery, the endometrium and peritoneal cavity invariably are contaminated with large numbers of highly pathogenic aerobic and anaerobic bacteria. The serosanguineous fluid that collects in the abdomen after surgery and the injured uterine tissue at the site of the incision provide excellent culture media for microbial growth. The bacterial inoculum is particularly large when cesarean section is performed after multiple vaginal examinations and extended duration of labor and ruptured membranes. ${ }^{5}$

Postoperative surgical site infection remains a major source of illness and a less frequent cause of death in the surgical patient ${ }^{6 .}$ Surgical site infection(SSI) is a common post operative complication and causes significant morbidity and mortality, increased antibiotic usage, prolonged hospital stay, adds cost and decreases patients quality of life.An estimated $40-60 \%$ of SSI are preventable with appropriate use of prophylactic antibiotics. However overuse, under use, improper use and misuse of antibiotics occurs in $25 \%-50 \%$ of operations $^{7}$. Patients who develop SSI's are up to $60 \%$ more likely to spend time in an ICU, 5 times more likely to be readmitted to the hospital, and 2 times more likely to die than are patients without an SSI .

Antibiotics administered prior to the contamination of previously sterile tissues or fluids are deemed 'prophylactic antibiotics'. Prevention of surgical site infection is the major goal of antibiotic prophylaxis ${ }^{1}$.

Rationale for using prophylactic antibiotics steps from very old familiar saying "prevention is better than cure". For optimal prevention of postoperative wound infection, it is necessary to follow a series of general principles. This includes the type of surgical intervention, class and character of antibiotic used, its time and route of administration ${ }^{8}$. 
Since infection by gram positive or negative organisms is possible hence antibiotic cover will be sufficiently comprehensive and effective if antimicrobial agent chosen has a wide spectrum of action ${ }^{8}$. The use of antibacterial prophylaxis of postoperative infection is firmly established within clean contaminated procedures. For clean procedures, prophylaxis has traditionally been reversed for operation involving foreign body implantation'. The administration of prophylactic antibiotics with in specific interval has been shown to reduce the burden of SSI, but adherence to proper timing guidelines remains problematic ${ }^{10}$.

The review of trials found evidence that prophylactic antibiotic in women undergoing caesarean section substantially reduced the incidence of episodes of fever, endometritis, wound infection, urinary tract infection and serious infection after caesarean section ${ }^{11}$. Prophylaxis is indicated for operations in which bacterial contamination is encountered or expected ${ }^{12}$. The therapeutic tissue levels of antibiotics should exist before the incision is made and the only exception is caesarean section where a special foetal consideration exists as the prophylactic drug masks neonatal sepsis ${ }^{13}$.

Preoperative antimicrobials can prevent infection when gives after cord clamping in emergency caesarean section, in high risk situation such as active labour or premature rupture of membranes ${ }^{14}$. Obesity appears to a risk factor of particular importance for wound infection ${ }^{15}$. Prophylaxis in low risk patients remains controversial. It increases adverse effects and costs. But a systematic literature review has shown that prophylaxis was beneficial following both elective and non-elective caesarean section. But USA surgical guidelines do not recommend routine prophylaxis in low risk patients ${ }^{14}$ The drug remains effective for $3-4$ hours. Depending on the agent selected, 1-3 doses are required i.e. coverage for first 24 hours. Overuse of prophylactic antibiotics lead to the development of resistance ${ }^{1}$. The ideal prophylactic antibiotic should be inexpensive, nontoxic, and active against some pathogens known to be involved and proven effective in clinical trials. It should not be a drug ordinarily used for therapy of an established infection ${ }^{13}$.

Approximately $30-50 \%$ of antibiotic use in hospitals is now for surgical prophylaxis. However $30-90 \%$ of this prophylaxis is inappropriate that increases the selective pressure favoring the emergence of antimicrobial resistance. Judicious use of antibiotics in the hospital through effective antibiotic policy and guideline development is then essential ${ }^{16}$.

\section{Antibiotic Prophylaxis}

HISTORICAL ASPECTS: Since the time chemotherapeutic drugs and antibiotics are available, they have been administered following clean surgery to reduce infectious morbidity ${ }^{13}$. Richards in 1943 evaluated the use of sulphonamides in certain gynecological operations. Haseltine \& Thelen in 1946 used sulphonamides as a prophylactic agent at cesarean section. Confusing \& heated debate concerning the efficacy of prophylactic antibiotics in surgery followed the publication of clinical trials during the 1950s. Keetle \& Plass in 1959 administered prophylactic penicillin to obstetric patients ${ }^{6,13}$.

Experimental studies published during the early 1960s helped clarify many of these problems and resulted in a more scientifically accurate approach to antimicrobial prophylaxis. Most important was the report by Burke, which demonstrated the crucial relationship between timing of antibiotic administration and its prophylactic efficacy. His experimental studies showed that to greatly reduce experimental skin infection produced by penicillin -sensitive S.aureus, the penicillin had to be in the skin shortly before or at the time of bacterial exposure. This study and others fostered the attitude that to prevent subsequent infection the antibiotic must be in the tissues before or at the time of bacterial contamination ${ }^{6,13}$.

As early as 1964, Bernard Cole reported on the successful use of prophylactic antibiotics in a randomized, prospective, placebo-controlled clinical study of abdominal operations on the gastrointestinal tract. The success of antibiotic prophylaxis noted in this early study was clearly due to the authors' appropriate patient selection and wise choice of available agents, as well as the timing of administration. Further advances in understanding of antibiotic prophylaxis in abdominal surgery occurred in 1970s. During this decade , the qualitative and quantitative nature of the endogenous flora in health and diseases was appropriately defined. Many prospective , blinded clinical studies in the 1980s prompted definitive recommendations concerning the proper approaches to antibiotic prophylaxis in surgery ${ }^{6,13}$.

BACKGROUND: Infection of the incised skin or soft tissue is a common but potentially avoidable complication of any surgical procedure. Some bacterial contamination of a surgical site is inevitable , either from the patient's own bacterial flora or from the environment ${ }^{18}$. Prophylactic use of antibiotics effectively reduces post operative infection. Prophylactic antibiotics are those that are administered to the patients before contamination has occurred. Optimal prophylaxis ensures that adequate concentrations of an appropriate antimicrobial are present in the serum, tissue and wound during the entire time that the incision is open and at risk for bacterial contamination ${ }^{19}$.

Wound infections are among the most common nosocomial infections among hospitalized surgical patients and are the second or third most common nosocomial infections among all hospitalized patients 
Surgical wound infections result in the administration of more antibiotics, an increase in the cost of care, and a prolongation of hospital stay. The aim of antibiotic prophylaxis is to prevent or at least decrease the incidence of postoperative wound infections ${ }^{20}$. Additional antibiotic use increases environmental pressure favoring the emergence of antimicrobial resistance. Extensive medical literature documents that the appropriate preoperative prophylactic use of antimicrobial agents can reduce the incidence of postoperative wound infections.

\section{Rationale}

Antibiotic prophylaxis is intended to reduce the size of bacterial inoculum and to change the characteristics of the culture medium at the operative site during the brief time that host defences are impaired by the trauma of surgery ${ }^{5}$. Three clinical criteria should be fulfilled to justify perioperative use of prophylactic antibiotics. First, the surgical procedure, by necessity, must be performed through a contaminated operative field. Second, their must be a high incidence of postoperative infections in the absence of prophylaxis. Finally, sequelae that may result from the primary infection must be significant ${ }^{5}$.

Most cesarean deliveries fulfill these criteria. The major objective in using prophylactic antibiotic is to reduce the incidence of post-cesarean endometritis. A second objective of prophylaxis is to reduce the incidence of these life threatening complications. The third objective of prophylaxis is to reduce the incidence of major wound infections ${ }^{5}$. Wound infection is more likely than endometritis to result in marked prolongation of the patients hospitalization.

\section{Choice Of Antibiotics}

The choice of a particular regimen is the product of scientific principles, practical considerations, empiricism and $\operatorname{cost}^{15}$. Although more than 20 different drugs have been used alone or in combination for antimicrobial prophylaxis during cesarean delivery ${ }^{4}$. Most obstetricians currently use either penicillin or a cephalosporin. One obvious principle is that the antibiotic should be active against organisms most likely to cause infection. The risk of postoperative febrile morbidity is reduced by a comparable amount by both broad spectrum penicillins \& cephalosporins. In one trial most striking risk reduction in postoperative febrile morbidity was achieved when a combination of broad spectrum penicillins and aminoglycosides were used for prophylaxis. The evidence from the trials in which broad spectrum penicillins and cephalosporins have been compared directly suggest that they have similar effects on the risks of postoperative febrile morbidity ${ }^{4}$.

Considering potential toxicity and cost, the least toxic, most effective, inexpensive antibiotic should be chosen. ${ }^{30}$. The choice of the antibiotic for prophylaxis is based on several factors. Always ask the patient about a prior history of antibiotic allergy, as beta-lactams are commonest type of antibiotics used in prophylaxis. A history of severe penicillin allergy means that cephalosporins are also contraindicated, as there is small but significant risk of cross reaction. ${ }^{19}$. Cephalosporins are widely favoured because of their broad spectrum activity and few side effects. Because first generation agents are more active against S.aureus are less expensive, and have a narrower spectrum of invitro activity, these agents are preferred for most surgical procedures.

\section{Recommended regimen: Cesarean section- Cefazolin 2g/iv-1 dose after cord clamping.}

GUIDELINES: ASHP therapeutic guideline Recommendation for antibiotic prophylaxis in cesarean section:The recommended regimen for all women (low and high risk) undergoing cesarean delivery is a single dose of cefazolin $2 \mathrm{~g}$ (as the sodium) intravenously immediately after clamping of the umbilical cord. ${ }^{4}$

ACOG practice bulletin considers the use of prophylaxis to be controversial in low-risk patients. ACOG does not routinely recommend prophylaxis in low-risk patients because of concerns about adverse effects, development of resistant organisms, and relaxation of standard infection-control measures and proper operative technique.

\section{Goals Of Antibiotic Prophylaxis}

* The goals of prophylactic administration of antibiotics to surgical patients are to:

* Reduce the incidence of surgical site infection.

* Use the antibiotic in a manner that is supported by evidence of effectiveness.

* Minimize the effect on patients normal flora

* Cause the minimal change to the patients host defences. ${ }^{18}$

It is important to emphasize that surgical antibiotic prophylaxis is an adjunct to, not a substitute for, good surgical technique. Antibiotic prophylaxis should be regarded as one component of an effective policy for the control of hospital acquired infection.

TIMING OF ADMINISTRATION: Timing of antibiotic administration is critical to its efficacy. The first dose should always be given before the procedure, preferably within 30 minutes before incision. ${ }^{19}$ The timing of administration is one of the most important aspects of prophylaxis regimens. Unlike other surgical procedures 
for which an antimicrobial is ideally administered just before incision, administration of antimicrobials in cesarean delivery is delayed after cord clamping. ${ }^{4}$ This is done principally to avoid suppression of the infants normal bacterial flora. Although toxicity in the infant is of potential concern, a majority of drugs used for this procedure have a documented record of safety in the treatment of infections during pregnancy, \& many are used in the treatment of neonatal sepsis. ACOG \& American Academy of Paediatrics recommend administration of prophylactic antibiotics after cord clamping. ${ }^{4}$

DURATION: Persistence of tissue concentrations past the period of surgery and recovery of normal physiology following anaesthesia does not improve efficacy and increases toxicity and cost. If the operation lasts four hours or less, one antibiotic dose is usually sufficient. In prolonged surgery of greater than four hours , further antibiotic dose may be required to maintain the concentration, particularly if the antibiotic has a short half life . Antimicrobial dose should begin within 60 minutes before surgical incision and that prophylactic antimicrobial agents should be discontinued within 24 hours of surgery ${ }^{20}$. Prolonged use of prophylactic antimicrobials is associated with emergence of resistant bacterial strains.

ROUTE OF ADMINISTRATION: The oral route tends to suffer from variable absorption, especially in presence of anaesthetic pre-medication, and this also makes it unsatisfactory prophylactic antibiotics are usually given intravenously as a bolus on induction of anaesthesia to ensure adequate tissue concentrations at the time of surgical incision. ${ }^{19}$

ANTIMICROBIAL DOSING: There are limited published data on appropriate antimicrobial dosing for prophylaxis. The drug should be provided in an adequate dose on the basis of patient body weight, adjusted dosing weight, or body mass index , and administration should be repeated intra-operatively if the operation is still in progress 2 half- lives after the the first dose to ensure adequate antimicrobial levels until wound closure. Those patients receiving 2-g of cefazolin had an incidence of SSI that was lower than that among those receiving $1-\mathrm{g}$ dose. ${ }^{20}$

COST: Pharmacoeconomic studies have been lacking or inadequate with regard to prophylactic use of antimicrobials; therefore a cost minimization approach was employed in developing these guidelines. When antimicrobials have been shown to be equally efficacious and safe, the recommendation is based on the least expensive agent.The other antimicrobials are considered to be alternative agents. The recommendation of an antimicrobial is determined primarily by efficacy and secondarily by cost. Because of variations in cost from one health system to another, health systems must tailor the choice of antimicrobials to their individual acquision costs. ${ }^{4}$

\section{References}

[1]. Joseph .T.Dipiro. PHARMACOTHERAPY. A pathophysiologic approach. $5^{\text {th }}$ edition , 1999. page 2111-2120.

[2]. Hugh RK.Barder,Sherwin A Kautman. Quick Reference To OB-GYN Procedures. III edition, 1990.page:113-18.

[3]. Thigpen BD, Hood WA, Chauhan S, Bufkin L, Bofill J, Magann E, Morrison JC.Timing of prophylactic antibiotic administration in the uninfected laboring gravida: a randomized clinical trial. Am J Obstet Gynecol. 2005 Jun;192(6):1864-8; discussion 1868-71

[4]. American society of health system pharmacists.ASHP therapeutic guidelines on antimicrobial prophylaxis in surgery.Am $\mathbf{J}$ Health Syst Pharm 1999; 56:1839-88.

5]. Clinical obstetrics by Carl.j.pauerstein.1987. page :-906-909.

[6]. P Ronald lee Nichols;Preventing surgical site infection : A surgeons perspective, special issue .EMERGING INFECTIOUS DISEASES. Available from: URL: http://www.cdc.gov/ncidod/EID/eid htm.

[7]. Nongyao Kasatpibal, RN, Silom Jamulitrat, MD, and Virasakthi Chongsuvivatwong, MD, PhD,for the surgical site infection study group.Hat Yai, Songkhla, Thailand. Standardized incidence rates of surgical site infection : a multicentre study in Thailand (AMJ infect control 2005; 33; 587-94).

[8]. Dikshit A, Gurubachan,Pandey SP, Shukla VK.Single dose oral cefpodoxime proxefil as antimicrobial prophylaxis in surgical procedures. The Indian practitioner . Nov 2003, vol 56 no: 11 page-738-41.

[9]. De Lalla F. Surgical prophylaxis in practice.J hosp Infect 2002 jan ; 50 suppl A : S9-12

[10]. J A Tan, V N Naik and L Lingard. Exploring obstacles to proper timing of prophylactic antibiotics for surgical site infections Quality and Safety in Health Care 2006;15:32-38.

[11]. Antibiotic prophylaxis for cesarean section the Cochrane Database of systematic reviews 2006 issue-3. Published by john Wiley and sons 2001 .

[12]. Bohnen J. Principles of perioperative antibacterial prophylaxis management of surgical site infections. J Disease Management \& Health outcomes, Vol 6, no-2, August 1999, pp.73-81(9).

[13]. P Usha Krishna, D.K.Tanik, Pregnancy at risk current concept-4 $4^{\text {th }}$ edition. 1995. page-487-493.

[14]. John.E.Conte. Manual of Antibiotics and infectious diseases Treatment and prevention $9^{\text {th }}$ edition,2002 .page:88-93.

[15]. Enkin E tal . Oxford medical publications. A guide to effective care in pregnancy and child birth. Page-258-263.

[16]. Survey and evaluation of antibiotic prophylaxis usage in surgery wards of tertiary level institution before and after the implementation of clinical guidelines. INDIAN JOURNAL OF SURGERY original article, 2006, vol-68,Issue-3,page-150-156.

[17]. Goodman and Gilman, Hardman GJ, Limbird EL. The pharmacological basis of therapeutics. $9^{\text {th }}$ edition.1996.page-1091-99.

[18]. Antibiotic prophylaxis in surgery. SIGN publication; No-45.

[19]. Rogerwalker . Clinical pharmacy and therapeutics .III Edition,2003. page-569-580.

[20]. Antimicrobial prophylaxis for surgery. An advisory statement from the national surgical infection prevention project. Clinical infectious diseases. 2004; 38, 1706-15. 